\title{
Acarbose Treatment and the Risk of Cardiovascular Disease in Type 2 Diabetic Patients: A Nationwide Seven-Year Follow-Up Study
}

\author{
Jui-Ming Chen, ${ }^{1,2,3}$ Cheng-Wei Chang, ${ }^{4}$ Ying-Chieh Lin, ${ }^{2}$ \\ Jorng-Tzong Horng, ${ }^{2,5}$ and Wayne H.-H. Sheu, ${ }^{6,7,8,9}$ \\ ${ }^{1}$ Department of Endocrinology and Metabolism, Tungs' Taichung MetroHarbor Hospital, Taichung 435, Taiwan \\ ${ }^{2}$ Department of Biomedical Informatics, Asia University, Taichung 413, Taiwan \\ ${ }^{3}$ Department of Gerontechnology and Service Management, Nan Kai University of Technology, Nantou 542, Taiwan \\ ${ }^{4}$ Department of Information Management, Hsing Wu University, New Taipei City 244, Taiwan \\ ${ }^{5}$ Department of Computer Science and Information Engineering, National Central University, Chungli 320, Taiwan \\ ${ }^{6}$ Division of Endocrinology and Metabolism, Department of Internal Medicine, Taichung Veterans General Hospital, \\ 160, Section 3, Taichung-Harbor Road, Taichung 407, Taiwan \\ ${ }^{7}$ School of Medicine, National Defense Medical Center, Taipei 114, Taiwan \\ ${ }^{8}$ School of Medicine, National Yang-Ming University, Taipei 112, Taiwan \\ ${ }^{9}$ Institute of Medical Technology, National Chung-Hsing University, Taichung 402, Taiwan
}

Correspondence should be addressed to Jorng-Tzong Horng; horng@db.csie.ncu.edu.tw and Wayne H.-H. Sheu; whhsheu@vghtc.gov.tw

Received 30 April 2014; Accepted 14 June 2014; Published 7 July 2014

Academic Editor: Nikolaos Papanas

Copyright (C) 2014 Jui-Ming Chen et al. This is an open access article distributed under the Creative Commons Attribution License, which permits unrestricted use, distribution, and reproduction in any medium, provided the original work is properly cited.

Objective. To investigate the potential benefits of acarbose treatment on cardiovascular disease (CVD) in patients with type 2 diabetes by using nationwide insurance claim dataset. Research Design and Methods. Among 644,792 newly diagnosed type 2 diabetic patients without preexisting CVD in a nationwide cohort study, 109,139 (16.9\%) who had received acarbose treatment were analyzed for CVD risk. Those with CVD followed by acarbose therapy were also subjected to analysis. Result. During 7 years of follow-up, 5,081 patients (4.7\%) developed CVD. The crude hazard ratio (HR) and adjusted HR were 0.66 and 0.99 , respectively. The adjusted HR of CVD was 1.19, 0.70, and 0.38 when the duration of acarbose use was $<12$ months, $12-24$ months, and $>24$ months, respectively. Adjusted HR was 1.14, 0.64, and 0.41 with acarbose cumulative doses $<54,750 \mathrm{mg}, 54,751$ to $109,500 \mathrm{mg}$, and $>109,500 \mathrm{mg}$, respectively. Conclusion. In patients with type 2 diabetes without preexisting CVD, treatment with acarbose showed a transient increase in incidence of CVD in the initial 12 months followed by significant reductions of CVD in prolonged acarbose users. After the first CVD events, continuous use of acarbose revealed neutral effect within the first 12 months. The underlying mechanisms require further investigations.

\section{Introduction}

Previous observational studies have indicated that postprandial hyperglycemia seems to play a unique role in the development of cardiovascular complications in patients with type 2 diabetes [1-4]. This is particularly relevant for Asian diabetic subjects whose postprandial hyperglycemia contributes more prominently to the level of HbAlc [5].
Acarbose, an oral antidiabetic agent, competitively inhibits the alpha-glucosidase in the brush-border of the small intestine and delays the digestion of complex carbohydrates in the upper small bowel that subsequently retards the absorption of glucose and blunts the postprandial hyperglycemia $[6,7]$. Compared to metformin, acarbose is similar in efficacy to initial therapy in Chinese patients newly diagnosed with type 2 diabetes [8]. Results from the STOP 


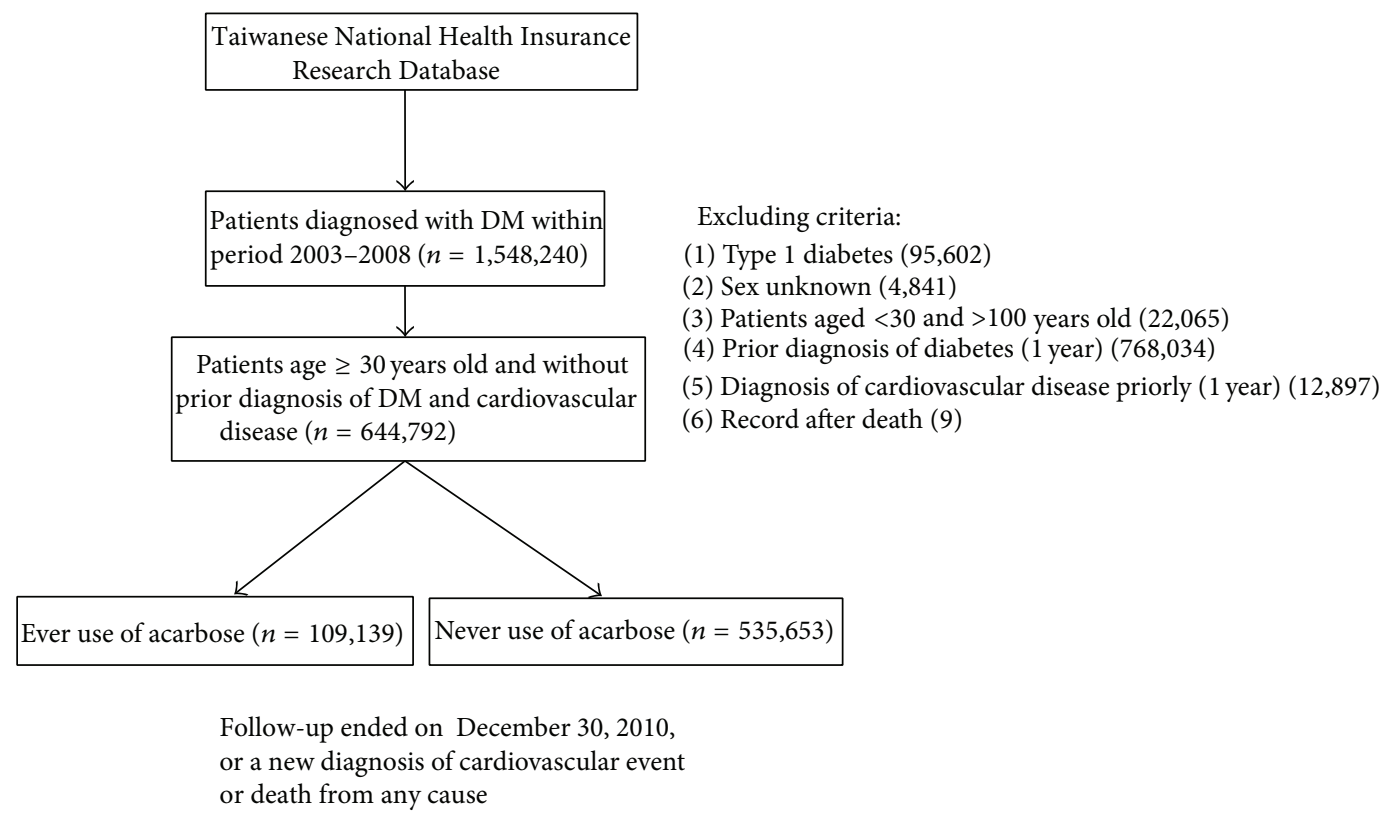

FIGURE 1: Study subjects' selection flow chart in those newly diagnosed with diabetes who do not have preexisting CVD.

NIDDM (Study to Prevent Non-Insulin-Dependent Diabetes Mellitus) had shown that acarbose treatment was associated with a $49 \%$ risk reduction in cardiovascular events in subjects with impaired glucose tolerance [9]. Even after adjusting for major risk factors, the reduction in the risk of cardiovascular events and hypertension associated with acarbose treatment was still statistically significant. In a meta-analysis of patients with type 2 diabetes, acarbose use was associated with a $35 \%$ reduction in cardiovascular events [10]. The mechanisms by which acarbose lowers the risk of cardiovascular events is putatively attributed to the diminution in postprandial hyperglycemic excursion and thus leads to a lowering of the impact of oxidative stress and tissues protection as well as a series of benefits $[6,11]$.

However, the above-mentioned findings were either limited to use in subjects with IGT (impaired glucose tolerance) [9], short-term studies [11], or meta-analysis [12]. Currently, there are no population-based long-term investigations about the potential cardiovascular effect of acarbose treatment in patients with type 2 diabetes. To address this issue, we used the Taiwanese National Health Insurance Research Database (NHIRD) to evaluate the cardiovascular effect of acarbose treatment in newly type 2 diabetic patients without previous CVD [13]. We also examined the effects of continuous use of acarbose on recurrent CVD in those diabetic subjects who had first cardiovascular events.

\section{Research Design and Methods}

2.1. Data Source. Since March 1, 1995, Taiwan had launched a single-payer National Health Insurance Program.

And in 2007, 98\% of Taiwan's populations had been enrolled in this program [13]. Data in the National Health Insurance Research Database (NHIRD) that could be used to identify patients or care providers is scrambled before being sent for database construction. The database is further scrambled before being released to researchers [13].

2.2. Methods. In this study, diabetic patients were identified using the ICD-9-CM code (250) with at least 3 outpatient visits or hospitalization treatment once which has been validated. Diabetic subjects were enrolled if they fulfilled the following three criteria: (1) diagnosed with diabetes between year 2003 and 2008; (2) age older than 30 years and less than 100 years as of January 1, 2003; and (3) had no diagnosis of cardiovascular diseases before year 2003 or one year prior to the diagnosis of diabetes.

Patients with type 1 diabetes, having diagnosis of diabetes in prior one year, and with sex unknown in the data were all excluded. The primary endpoint is cardiovascular diseases described below. The follow-up period started from Jan. 1, 2003 and lasted until Dec. 31, 2010 (Figure 1).

Cardiovascular outcomes were identified with the diagnosis of cardiovascular disease (coronary heart disease with ICD-9-CM codes 410-414, stroke with ICD-9CM codes 430438 , and peripheral arterial occlusive disease (PAOD) with ICD-9CM codes 443.9). To confirm the diagnosis, all above events required hospitalization for at least once.

The comorbidities, including hypertension (ICD-9CM codes 401-405), chronic kidney disease (CKD) (ICD-9CM codes 582-583), and hyperlipidemia (ICD-9CM codes 272), were recorded with at least 3 times of outpatient visit or admission once.

Using the NHIRD, a total of 644,792 newly diagnosed type 2 diabetic patients were enrolled. Among them 109,139 had received acarbose treatment during the period of followup. The follow-up period ended on December 30, 2010 or when a newly diagnosed cardiovascular event or death from any cause developed (Figure 1 and Table 1). 
TABLE 1: Demographics, comorbidities, and concomitant use of antidiabetic medications in those acarbose users with $(n=5081)$ or without (104058) CVD development.

\begin{tabular}{|c|c|c|c|c|}
\hline Characteristic & $\begin{array}{c}\text { Overall number } \\
109,139 \\
\end{array}$ & $\begin{array}{c}\text { Did not develop CVD } \\
104,058 \\
\end{array}$ & $\begin{array}{c}\text { Developed CVD } \\
5,081 \\
\end{array}$ & $P$ value \\
\hline \multicolumn{5}{|c|}{ Age, years $($ mean $\pm S D)$} \\
\hline $30-39$ & $9,417(8.6)$ & $9,239(8.9)$ & $178(3.5)$ & \multirow{7}{*}{$<0.0001$} \\
\hline $40-49$ & $25,707(23.6)$ & $24,936(24.0)$ & $771(15.2)$ & \\
\hline $50-59$ & $32,921(30.2)$ & $31,496(30.3)$ & $1,425(28.0)$ & \\
\hline $60-69$ & $22,343(20.5)$ & $21,035(20.2)$ & $1,308(25.7)$ & \\
\hline $70-79$ & $14,295(13.1)$ & $13,269(12.8)$ & $1,026(20.2)$ & \\
\hline $80-89$ & $4,152(3.8)$ & $3,805(3.7)$ & $347(6.8)$ & \\
\hline$>=90$ & $304(0.3)$ & $278(0.3)$ & $26(0.5)$ & \\
\hline \multicolumn{5}{|l|}{ Sex } \\
\hline Male & $59,473(54.5)$ & $56,033(53.8)$ & $3,440(67.7)$ & \multirow{2}{*}{$<0.0001$} \\
\hline Female & $49,666(45.5)$ & $48,025(46.2)$ & $1,641(32.3)$ & \\
\hline \multicolumn{5}{|l|}{ Comorbidities } \\
\hline Hypertension & $81,341(74.5)$ & $76,944(73.9)$ & $4,397(86.5)$ & $<0.0001$ \\
\hline Hyperlipidemia & $70,106(64.2)$ & $67,213(64.6)$ & $2,893(56.9)$ & $<0.0001$ \\
\hline CKD & $6,965(6.4)$ & $6,348(6.1)$ & $617(12.1)$ & $<0.0001$ \\
\hline \multicolumn{5}{|c|}{ Other diabetes medications } \\
\hline Rosiglitazone & $9,420(8.6)$ & $9,010(8.7)$ & $410(8.0)$ & $<0.0001$ \\
\hline Metformin & $72,378(66.3)$ & $70,037(67.3)$ & $2,341(46.1)$ & $<0.0001$ \\
\hline Pioglitazone & $14,963(13.7)$ & $14,557(14.0)$ & $406(8.0)$ & $<0.0001$ \\
\hline Sulfonylurea & $51,765(47.4)$ & $49,915(48.0)$ & $1,850(36.4)$ & $<0.0001$ \\
\hline Meglitinide & $13,161(12.1)$ & $12,551(12.1)$ & $610(12.0)$ & $<0.0001$ \\
\hline Insulin & $8,994(8.2)$ & $8,426(8.1)$ & $568(11.2)$ & $<0.0001$ \\
\hline DPP4 inhibitor & $11,641(10.7)$ & $11,528(11.1)$ & $113(2.2)$ & $<0.0001$ \\
\hline
\end{tabular}

CVD: cardiovascular disease. DPP4: dipeptidyl peptidase 4. CKD: chronic kidney disease.

Cumulative duration of taking acarbose was calculated by counting the number of days between prescriptions. If the next prescription was filled within 30 days of the expected end date of the previous prescription, we assumed that therapy was uninterrupted. However, if there were no refills within the 30 days after the expected end date of the previous prescription, we assumed a gap in therapy starting 30 days after the date that the previous prescription should have ended. The cumulative duration variable was a time-varying sum of all periods of exposure even if there were gaps in treatment. Cumulative dose of acarbose was calculated in a similar fashion.

Among the 5081 patients who had received acarbose and developed CVD, 456 patients who died in the first month were excluded. A total of 4625 patients were enrolled in this study and followed up to record their recurrent CVD events. Among them, 1756 patients kept acarbose therapy while 2869 patients stopped receiving acarbose and switched to other antidiabetic regimens (see Supplemental Figure 1s of the Supplementary Material available online at http://dx.doi.org/10.1155/2014/812628).

The protocols had been submitted to the Tungs' Taichung MetroHarbor Hospital IRB (institutional review board) for review and approval was obtained.
2.3. Statistical Analysis. Distributions of subjects with and without use of acarbose according to age, gender, clinical comorbidities, and concomitant use of other diabetes medications were examined using $\chi^{2}$-tests for categorical variables and Student's $t$-tests for continuous variables. The crude, age, gender, use of other diabetes medications, CharlsonDeyo comorbidity index (CCI), adjusted hazard ratio, and 95\% confidence interval were calculated in subjects with and without use of acarbose, respectively. All analyses were performed using the SAS software, version 9.2 (SAS Institute, Cary, NC, USA).

\section{Results}

Among 644,792 patients with newly diagnosed type 2 diabetes identified during 2003 to 2008, 109,139 (16.9\%) were treated with acarbose, either as monotherapy or in combination with other antidiabetic regimens. As compared with nonusers of acarbose, the acarbose users were younger, slightly female predominant, had more comorbidities, and were more likely to take combination treatment with other antidiabetic regimens. During 7 years (median 3.8 years) of follow-up, 5081 patients (4.7\%) who had received acarbose treatment developed CVD as compared to 33,203 patients 
TABLE 2: Crude and adjusted hazard ratio of CVD with or without acarbose use.

\begin{tabular}{|c|c|c|c|c|c|c|c|c|c|c|}
\hline \multirow{3}{*}{ Risk factor } & \multirow{3}{*}{ Developed CVD } & \multirow{3}{*}{ Did not develop CVD } & \multicolumn{3}{|c|}{ Crude } & \multicolumn{5}{|c|}{ Adjusted } \\
\hline & & & \multirow{2}{*}{$\mathrm{HR}$} & \multicolumn{2}{|c|}{$95 \% \mathrm{CI}$} & \multirow[t]{2}{*}{$P$} & \multirow{2}{*}{ HR } & \multicolumn{2}{|c|}{$95 \%$ CI } & \multirow[t]{2}{*}{$P$} \\
\hline & & & & Low & Up & & & Low & Up & \\
\hline Never use of acarbose & 33203 & 502450 & Ref. & - & - & & Ref. & - & - & - \\
\hline Ever use of acarbose & 5081 & 104058 & 0.66 & 0.641 & 0.680 & $<0.001$ & 0.99 & 0.958 & 1.019 & 0.443 \\
\hline \multicolumn{11}{|c|}{ Cumulative duration of therapy (months) } \\
\hline$<12$ & 4216 & 69976 & 0.83 & 0.802 & 0.855 & $<0.001$ & 1.19 & 1.152 & 1.231 & $<0.001$ \\
\hline $12-24$ & 545 & 17345 & 0.42 & 0.387 & 0.459 & $<0.001$ & 0.70 & 0.643 & 0.762 & $<0.001$ \\
\hline$>24$ & 320 & 16737 & 0.24 & 0.217 & 0.271 & $<0.001$ & 0.38 & 0.341 & 0.425 & $<0.001$ \\
\hline \multicolumn{11}{|l|}{ Cumulative dose (mg) } \\
\hline $1-54,750$ & 4373 & 75757 & 0.79 & 0.769 & 0.819 & $<0.001$ & 1.14 & 1.105 & 1.18 & $<0.001$ \\
\hline $54,751-109,500$ & 449 & 15273 & 0.39 & 0.357 & 0.43 & $<0.001$ & 0.64 & 0.583 & 0.704 & $<0.001$ \\
\hline$>109,500$ & 259 & 13028 & 0.25 & 0.219 & 0.28 & $<0.001$ & 0.41 & 0.360 & 0.460 & $<0.001$ \\
\hline
\end{tabular}

Adjusted age, gender, other diabetic medications, and CCI. CVD: cardiovascular disease.

TABLE 3: HR of recurrent CVD by keep or stop using acarbose.

\begin{tabular}{|c|c|c|c|c|c|c|c|c|c|c|}
\hline \multirow{3}{*}{ Risk factor } & \multirow{3}{*}{ Recurrence of CVD } & \multirow{3}{*}{ No recurrence of CVD } & \multicolumn{3}{|c|}{ Crude } & \multicolumn{5}{|c|}{ Adjusted } \\
\hline & & & \multirow{2}{*}{$\mathrm{HR}$} & \multicolumn{2}{|c|}{$95 \%$ CI } & \multirow[t]{2}{*}{$P$} & \multirow{2}{*}{ HR } & \multicolumn{2}{|c|}{$95 \%$ CI } & \multirow[t]{2}{*}{$P$} \\
\hline & & & & Low & Up & & & Low & Up & \\
\hline Stop acarbose users & 627 & 2242 & Ref. & - & - & & Ref. & - & - & - \\
\hline Keep acarbose users & 582 & 1174 & 0.84 & 0.752 & 0.944 & 0.003 & 0.88 & 0.781 & 0.987 & 0.029 \\
\hline \multicolumn{11}{|c|}{ Cumulative duration of therapy (months) } \\
\hline$<12$ & 508 & 1142 & 0.98 & 0.87 & 1.1 & 0.715 & 1.00 & 0.891 & 1.132 & 0.947 \\
\hline $12-24$ & 49 & 18 & 0.52 & 0.392 & 0.702 & $<0.001$ & 0.48 & 0.355 & 0.645 & $<0.001$ \\
\hline$>24$ & 25 & 14 & 0.29 & 0.196 & 0.441 & $<0.001$ & 0.38 & 0.249 & 0.567 & $<0.001$ \\
\hline \multicolumn{11}{|l|}{ Cumulative dose (mg) } \\
\hline $1-10500$ & 523 & 1149 & 0.95 & 0.848 & 1.07 & 0.413 & 0.97 & 0.86 & 1.091 & 0.600 \\
\hline $10501-28000$ & 39 & 16 & 0.45 & 0.324 & 0.621 & $<0.001$ & 0.47 & 0.338 & 0.657 & $<0.001$ \\
\hline$>28000$ & 20 & 9 & 0.33 & 0.211 & 0.518 & $<0.001$ & 0.40 & 0.253 & 0.628 & $<0.001$ \\
\hline
\end{tabular}

Adjusted age, gender, other diabetic medications, and CCI. CVD: cardiovascular disease.

(6.2\%) who never took acarbose therapy. Furthermore, 2619 patients $(2.4 \%)$ who had received acarbose treatment developed stroke as compared to $18212(3.4 \%)$ who never took acarbose therapy. The study showed that acarbose users group had a lower risk of developing CVD $(P<0.001)$ and stroke $(P<0.037)$ (Supplemental Table 1s). Table 1 depicted data of those with or without CVD in acarbose users. Those with CVD were older, male predominant, had more comorbidities with hypertension and CKD but were less likely to have hyperlipidemia (all $P<0.001$ ), and had lesser use of combined oral hypoglycemic agents but more use of insulin as compared to those without CVD (Table 1).

By using those without exposure to acarbose as reference value, the crude ( $95 \% \mathrm{CI}$ ) hazard ratio (HR) of acarbose users who developed CVD was 0.66 (95\% CI 0.641-0.680, $P<$ 0.001 ) (Table 2). After adjusting for age, gender, CharlsonDeyo comorbidity index (CCI), and concomitant use of other antidiabetic regimens, the adjusted HR became 0.99 (0.958 to $1.019, P=0.443)$. Further analysis indicated that adjusted HR of CVD was 1.19 (95\% CI 1.152-1.231 $P<0.001)$, 0.70 (95\% CI $0.643-0.762 P<0.001$ ), and 0.38 (95\% CI $0.341-0.425$ $P<0.001)$ when the duration of acarbose use to CVD events was $<12$ months, $12-24$ months and $>24$ months, respectively. Adjusted HR was 1.14 (95\% CI 1.105-1.18 $P<0.001), 0.64$ (95\% CI $0.583-0.704 P<0.001$ ), and 0.41 (95\% CI 0.360 $0.460 P<0.001)$ with acarbose cumulative dose $<54,750 \mathrm{mg}$, 54,751 to $109,500 \mathrm{mg}$, and $>109,500 \mathrm{mg}$, respectively (Table 2).

For patients who had first event of CVD, continuous use of acarbose revealed neutral adjusted HR 1.00 (95\% CI 0.891$1.132 P=0.947)$ for recurrent CVD in those with use duration $<12$ months followed by lower adjusted HR in those with prolonged acarbose use as compared to those who stopped using acarbose (Table 3). Further analysis, based on cumulative dosages, showed a similar trend (Table 3 ).

\section{Discussion}

The main findings from the present dataset indicated that use of acarbose, either as monotherapy or in combination with other antidiabetic regimens, in newly diagnosed type 2 diabetes subjects who did not have preexisting CVD, provided a unique impact on the subsequent development of CVD. Specifically, use of acarbose increased the chances of developing CVD in the first 12 months of exposure. However, 
the benefits on CVD began to emerge in continuous users of acarbose. Analysis of dosages of acarbose showed similar patterns.

Results from previous observational studies have demonstrated that elevated postprandial glycemic excursion contributes to increase in the risk of developing coronary heart disease or a CV event $[14,15]$. By reducing postprandial hyperglycemia, the potential benefits derived from acarbose treatment have included reduced body weight $[9,12]$, reduced blood pressure both systolic and diastolic $[9,10,16,17]$, reduced triglyceride [18], reduced post meal lipemia [1921], reduced postmeal activation of coagulation [22], reduced insulin resistance $[18,23]$, reduced carotid intima-medial thickness [24], reduced myocardial infarct size [25], marked decrease in the plasma levels of plasminogen activator inhibitor 1 and fibrinogen, and reduction in the plasma levels of oxidized low-density lipoprotein [26]. In fact, findings from STOP NIDDM [9] in subjects with IGT and results from MeRIA study [12] all support the potential benefits of acarbose management on CVD.

The exact causes of our unanticipated findings that acarbose increased CVD in the first 12 months of exposure while providing protection in those prolonged users are not clear at this moment. Our target diabetic subjects were newly diagnosed with diabetes without preexisting CVD which was different from the subjects of STOP NIDDM [1] and MeRIA [12]. Considering the time needed to form atherosclerosis, it is not reasonable to speculate that the use of acarbose enhanced occurrence of CVD events. In this regard, there has also no evidence that acarbose could precipitate rupture of those preexisting vascular plaques in acarbose users. Actually, recent study indicated that the use of acarbose could rapidly improve carotid plaque echolucency within 1 month of therapy in type 2 diabetes patients with acute coronary syndrome [27]. On the other hand, diabetic subjects who used acarbose for a prolonged period or who reached certain amount of doses showed benefits of protection, both in first CVD or recurrent CVD events. The exact mechanisms definitely require further investigations.

There are several limitations in our study. First, a causal association between acarbose and CVD cannot be ascertained based on the observational data. We cannot retrieve clinical data like lipids and glycemic, blood pressure control from this claim dataset. Although we have adjusted potential confounding, there may be other measured and unmeasured factors which we were not able to detect or obtain. The diagnosis of type 2 diabetes or the cardiovascular events according to the ICD-9-CM may also lead to some distortion.

\section{Conclusions}

Our study suggests that treatment with acarbose in the newly diagnosed type 2 diabetic patients who do not have preexisting CVD showed an initial increased $\mathrm{HR}$ followed by benefits on subsequent CVD. After the first CVD events, continuous use of acarbose revealed a relatively similar pattern of benefits on developing recurrent CVD. The effects of acarbose on CVD remain speculative before the final results of other large prospective studies come out (such as the ongoing Acarbose Cardiovascular Evaluation Trial) [28].

\section{Conflict of Interests}

The authors declare that there is no conflict of interests regarding the publication of this paper.

\section{Authors' Contribution}

Jui-Ming Chen and Wayne H.-H. Sheu conceived and designed the experiments. Jui-Ming Chen, Jorng-Tzong Horng, and Cheng-Wei Chang performed the experiments. Jui-Ming Chen, Wayne H.-H. Sheu, Cheng-Wei Chang, and Ying-Chieh Lin analyzed the data. Jorng-Tzong Horng contributed to reagents/materials/analysis tools. Jui-Ming Chen, Wayne H.-H. Sheu, and Cheng-Wei Chang wrote the paper and contributed equally to this work.

\section{References}

[1] W. H. Sheu, A. Rosman, A. Mithal et al., "Addressing the burden of type 2 diabetes and cardiovascular disease through the management of postprandial hyperglycaemia: an Asian-Pacific perspective and expert recommendations," Diabetes Research and Clinical Practice, vol. 92, no. 3, pp. 312-321, 2011.

[2] L. P. Lowe, K. Liu, P. Greenland, B. E. Metzger, A. R. Dyer, and J. Stamler, "Diabetes, asymptomatic hyperglycemia, and 22-year mortality in black and white men: the Chicago Heart Association Detection Project in industry study," Diabetes Care, vol. 20, no. 2, pp. 163-169, 1997.

[3] "Glucose tolerance and mortality: comparison of WHO and American Diabetes Association diagnostic criteria. The DECODE study group. European Diabetes Epidemiology Group. Diabetes Epidemiology: collaborative analysis of Diagnostic criteria in Europe," The Lancet, vol. 354, pp. 617-621, 1999.

[4] T. Nakagami, "Hyperglycaemia and mortality from all causes and from cardiovascular disease in five populations of Asian origin," Diabetologia, vol. 47, no. 3, pp. 385-394, 2004.

[5] J.-S. Wang, S.-T. Tu, I.-T. Lee et al., "Contribution of postprandial glucose to excess hyperglycaemia in Asian type 2 diabetic patients using continuous glucose monitoring," Diabetes/Metabolism Research and Reviews, vol. 27, no. 1, pp. 79-84, 2011.

[6] E. Standl and O. Schnell, "Alpha-glucosidase inhibitors 2012cardiovascular considerations and trial evaluation," Diabetes and Vascular Disease Research, vol. 9, no. 3, pp. 163-169, 2012.

[7] H. Bischoff, "Pharmacology of $\alpha$-glucosidase inhibition," European Journal of Clinical Investigation, vol. 24, supplement 3, pp. 3-10, 1994.

[8] W. Yang, L. Jie, Z. Shan et al., "Acarbose compared with metformin as initial therapy in patients with newly diagnosed type 2 diabetes: an open-label, non-inferiority randomised trial," The Lancet Diabetes \& Endocrinology, vol. 2, no. 1, pp. 4655, 2014.

[9] J. Chiasson, R. G. Josse, R. Gomis, M. Hanefeld, A. Karasik, and M. Laakso, "Acarbose treatment and the risk of cardiovascular disease and hypertension in patients with impaired glucose tolerance: the STOP-NIDDM trial," Journal of the American Medical Association, vol. 290, no. 4, pp. 486-494, 2003. 
[10] N. Papanas and E. Maltezos, "Oral antidiabetic agents: antiatherosclerotic properties beyond glucose lowering?" Current Pharmaceutical Design, vol. 15, no. 27, pp. 3179-3192, 2009.

[11] J. S. Wang, S. D. Lin, W. J. Lee et al., "Effects of acarbose versus glibenclamide on glycemic excursion and oxidative stress in type 2 diabetic patients inadequately controlled by metformin: a 24-week, randomized, open-label, parallel-group comparison," Clinical Therapeutics, vol. 33, no. 12, pp. 1932-1942, 2011.

[12] M. Hanefeld, M. Cagatay, T. Petrowitsch, D. Neuser, D. Petzinna, and M. Rupp, "Acarbose reduces the risk for myocardial infarction in type 2 diabetic patients: meta-analysis of seven long-term studies," European Heart Journal, vol. 25, no. 1, pp. 10-16, 2004.

[13] NHIRD, Introduction to the National Health Insurance Research Database (NHIRD), Taiwan, 2013, http://nhird.nhri .org.tw/date_01.html.

[14] E. Standl, O. Schnell, and A. Ceriello, "Postprandial hyperglycemia and glycemic variability: should we care?" Diabetes Care, vol. 34, supplement 2, pp. S120-S127, 2011.

[15] M. Coutinho, H. C. Gerstein, Y. Wang, and S. Yusuf, “The relationship between glucose and incident cardiovascular events: a metaregression analysis of published data from 20 studies of 95,783 individuals followed for 12.4 years," Diabetes Care, vol. 22, no. 2, pp. 233-240, 1999.

[16] P. Rosenbaum, R. B. Peres, M. T. Zanella, and S. R. G. Ferreira, "Improved glycemic control by acarbose therapy in hypertensive diabetic patients: effects on blood pressure and hormonal parameters," Brazilian Journal of Medical and Biological Research, vol. 35, no. 8, pp. 877-884, 2002.

[17] D. Gentilcore, L. Vanis, J. M. Wishart, C. K. Rayner, M. Horowitz, and K. L. Jones, "The alpha $(\alpha)$-glucosidase inhibitor, acarbose, attenuates the blood pressure and splanchnic blood flow responses to intraduodenal sucrose in older adults," Journals of Gerontology A: Biological Sciences and Medical Sciences, vol. 66, no. 8, pp. 917-924, 2011.

[18] R. Rachmani, Y. Bar-Dayan, Z. Ronen, Z. Levi, I. Slavachevsky, and M. Ravid, "The effect of acarbose on insulin resistance in obese hypertensive subjects with normal glucose tolerance: a randomized controlled study," Diabetes, Obesity and Metabolism, vol. 6, no. 1, pp. 63-68, 2004.

[19] R. Scott, C. J. Lintott, P. Zimmet, L. Campbell, K. Bowen, and T. Welborn, "Will acarbose improve the metabolic abnormalities of insulin-resistant type 2 diabetes mellitus?” Diabetes Research and Clinical Practice, vol. 43, no. 3, pp. 179-185, 1999.

[20] S. Kado, T. Murakami, A. Aoki et al., "Effect of acarbose on postprandial lipid metabolism in type 2 diabetes mellitus," Diabetes Research and Clinical Practice, vol. 41, no. 1, pp. 49-55, 1998.

[21] S. Salman, F. Salman, I. Satman et al., "Comparison of acarbose and gliclazide as first-line agents in patients with type 2 diabetes," Current Medical Research and Opinion, vol. 16, no. 4, pp. 296-306, 2001.

[22] A. Ceriello, C. Taboga, L. Tonutti et al., "Post-meal coagulation activation in diabetes mellitus: the effect of acarbose," Diabetologia, vol. 39, no. 4, pp. 469-473, 1996.

[23] K. Nakamura, S. Yamagishi, T. Matsui, and H. Inoue, "Acarbose, an $\alpha$-glucosidase inhibitor, improves insulin resistance in fructose-fed rats," Drugs under Experimental and Clinical Research, vol. 31, no. 4, pp. 155-159, 2005.

[24] M. Hanefeld, J. L. Chiasson, C. Koehler, E. Henkel, F. Schaper, and T. Temelkova-Kurktschiev, "Acarbose slows progression of intima-media thickness of the carotid arteries in subjects with impaired glucose tolerance," Stroke, vol. 35, no. 5, pp. 1073-1078, 2004.

[25] S. Minatoguchi, Z. Zhang, N. Bao et al., "Acarbose reduces myocardial infarct size by preventing postprandial hyperglycemia and hydroxyl radical production and opening mitochondrial KATP channels in rabbits," Journal of Cardiovascular Pharmacology, vol. 54, no. 1, pp. 25-30, 2009.

[26] Y. Shinoda, I. Inoue, T. Nakano et al., "Acarbose improves fibrinolytic activity in patients with impaired glucose tolerance," Metabolism, vol. 55, no. 7, pp. 935-939, 2006.

[27] M. Hirano, T. Nakamura, J. Obata et al., "Early improvement in carotid plaque Echogenicity by Acarbose in patients with acute coronary syndromes," Circulation Journal, vol. 76, no. 6, pp. 1452-1460, 2012.

[28] R. Holman, D. Hu, and C. Y. Pan, Acarbose Cardiovascular Evaluation (ACE), http://www.dtu.ox.ac.uk/ace/. 


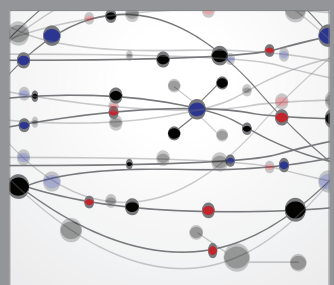

The Scientific World Journal
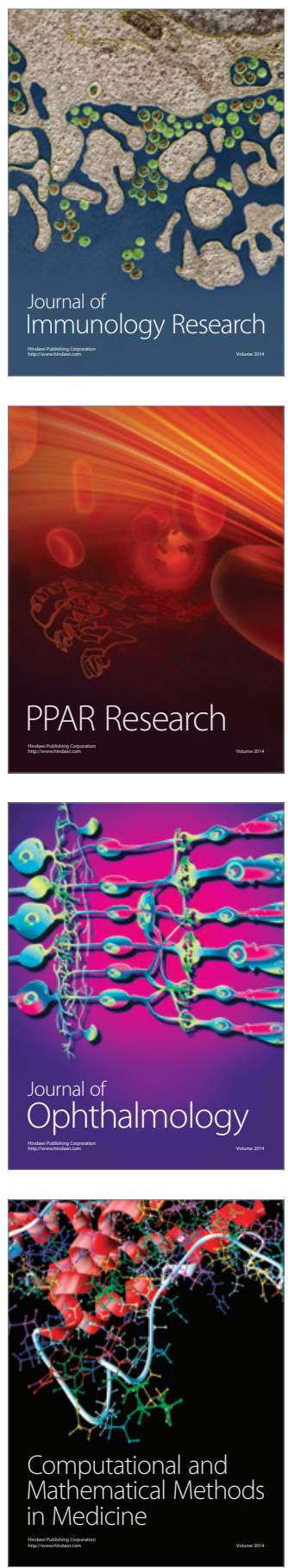

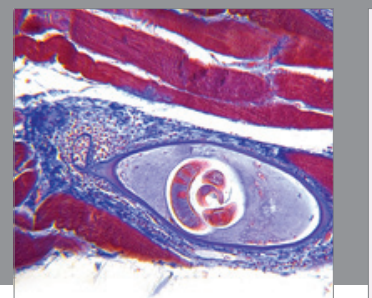

Gastroenterology

Research and Practice
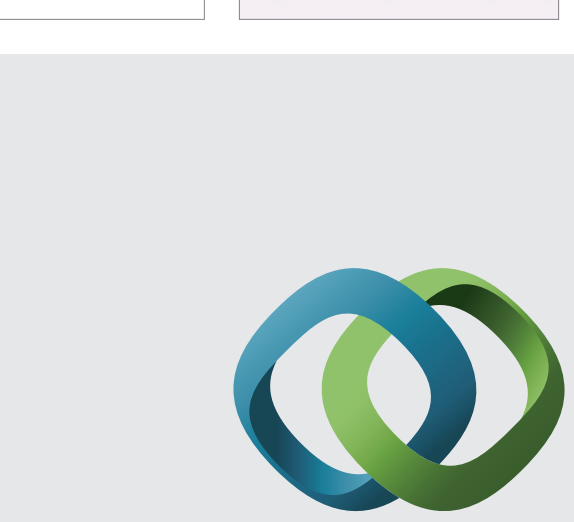

\section{Hindawi}

Submit your manuscripts at

http://www.hindawi.com
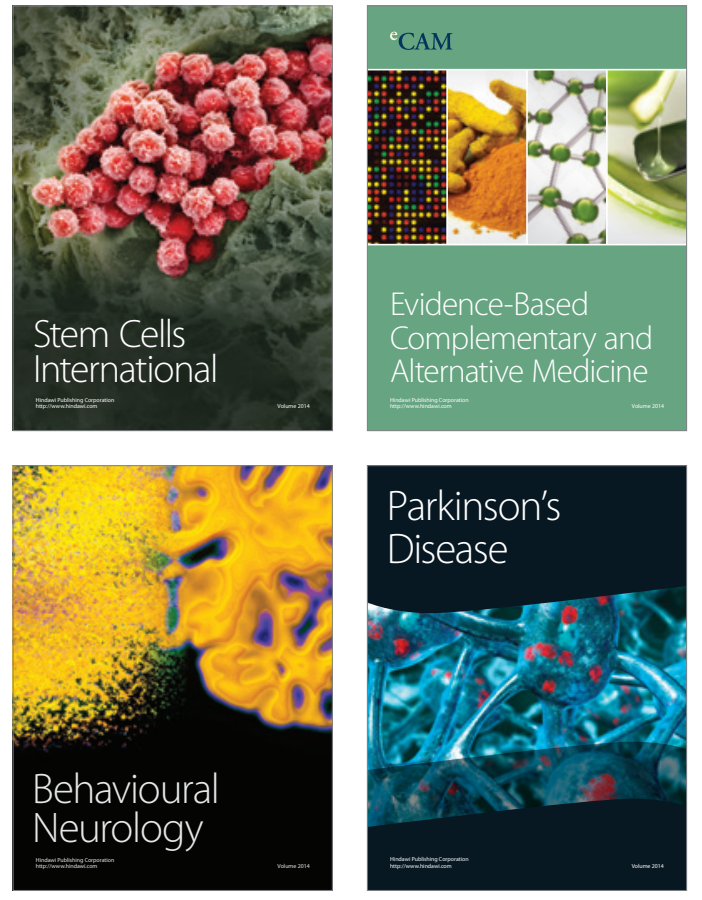
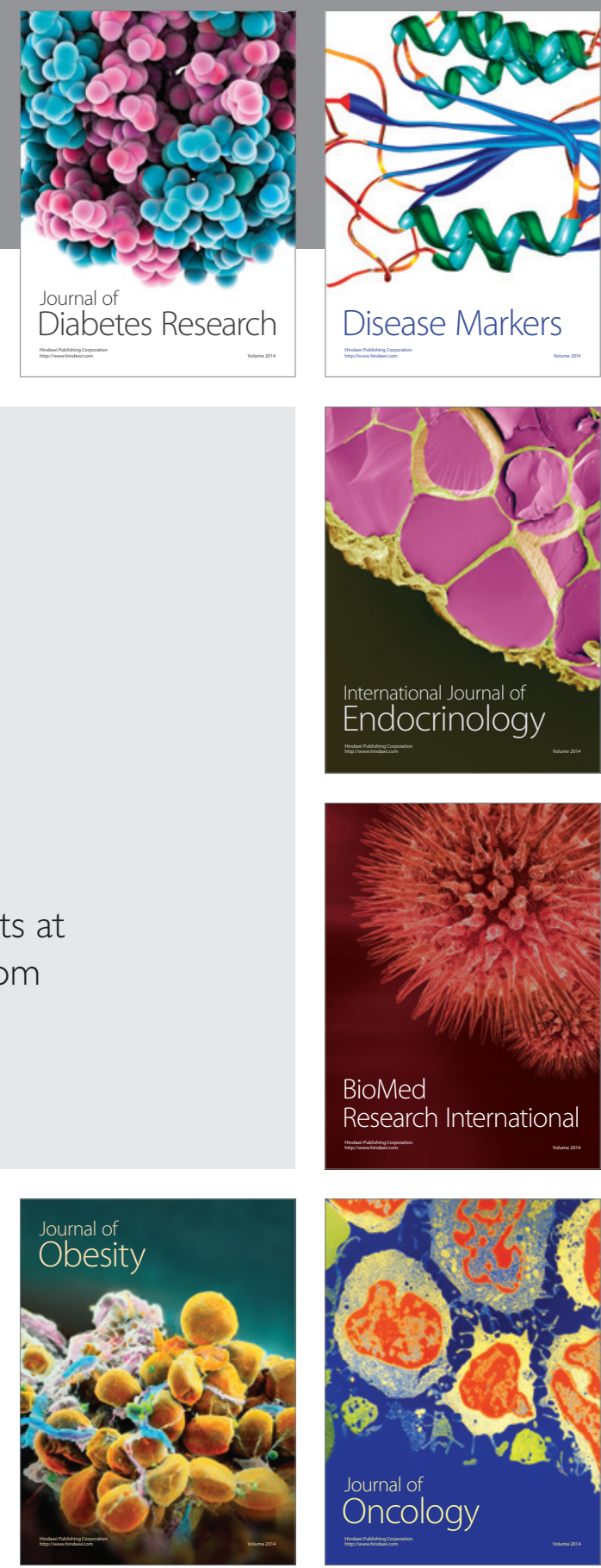

Disease Markers
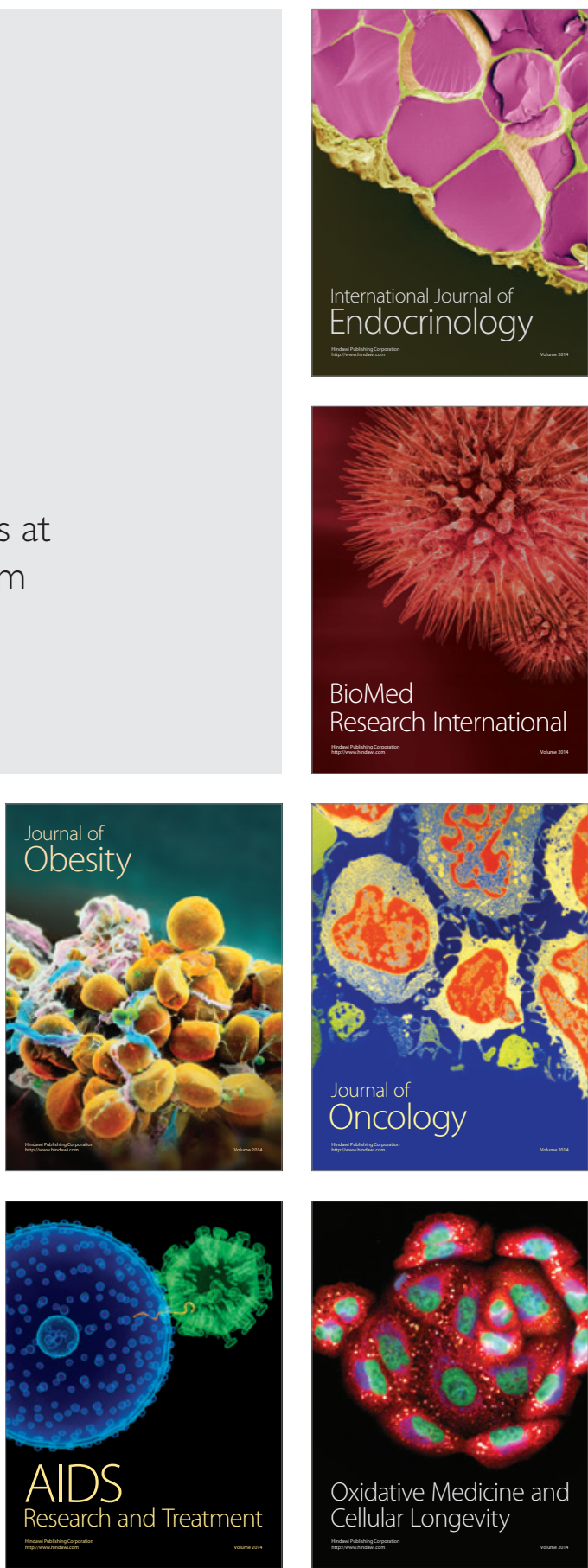